\title{
23
}

\section{On the semantics of cup}

Keith Allan

Like any language, a metalanguage for semantics consists of a set of symbols, a set of axioms and rules for combining them into syntactically well-formed structures, along with a set of interpretations for these structures and for the individual symbols in isolation. ${ }^{1}$ Human languages are the objects studied in semantics, consequently, the language under investigation is known as the 'object language'. The language that a linguist uses to describe and analyse the object language is called the 'metalanguage'. The basic requirement for a metalanguage is to satisfactorily communicate the meaning of item $e_{\mathrm{OL}}$-that is, any expression in the object language, whether it is a word, a phrase, a sentence, or (perhaps) a longer text-in terms of an expression ' $\mathrm{e}_{\mathrm{M}}$ ' in the metalanguage. A metalanguage is just another language, often an artificial and not a natural one. One important practical constraint on a metalanguage is that it needs to be understood by human beings who normally communicate in a natural language of which they have fluent command. If you understood neither Polish nor Swahili there is little point in my using Swahili as a metalanguage for the semantic analysis of Polish (or vice versa); for example, to say To jest pies means 'Ni mbwa' will not help you at all. Readers of this chapter must, perforce, know English, so we can use English as a metalanguage

1 Anna Wierzbicka is an exceptionally brilliant scholar who, during the last half-century, has generated a vast quantity of excellent published work on semantics for several languages. Furthermore, as this volume demonstrates, she has inspired an impressive number of followers who are fine scholars in their own right. Although my essay is critical of some aspects of Anna's semantic theory, it is an argument for a different point of view and assuredly NOT an attack on the high esteem with which Anna Wierzbicka is very properly held. 
and say To jest pies (in Polish) means 'It's a dog'; or we can say To jest pies means 'Ni mbwa' in Swahili, which means 'It's a dog'-here using English as a meta-metalanguage. As we see, the metalanguage is in effect a translation of the object language (cf. Carnap 1937: 228); in order for the metalanguage to be understood and used by human beings it must be communicable, and hence translate into a natural language. We must conclude that a metalanguage expression ' $\mathrm{e}_{\mathrm{M}}$ ' used in the semantic definition of a natural language expression $e_{\mathrm{OL}}$ will always be equivalent to the natural language expression through which it is interpreted.

The metalanguage is the language of a semantic theory. The principal function of the theory is to explain data (words, sentences) from natural language. The goal of the theory is to explain all the data that it was constructed to explain; therefore, limitations on its range need to be clearly stated. A theory should have predictive power insofar as it raises expectations about data that have not yet come to light. It is absolutely necessary that a theory be internally consistent. But what about its external relations? No theory of semantics can completely ignore syntax and phonology, and the ideal semantic theory will integrate with theories of both these components of a grammar. Semantic theory should also integrate with theories of pragmatics that seek to explain meaning in social and cultural contexts and with theories of discourse structure. A semantic theory should not only make useful revelations about the nature of human language but also about human cognition because meaning is often a reflex of human perception and conception. All theories, without exception, are abstractions from reality; so the relation of theory to reality 'is not analogous to that of soup to beef but rather of check number and overcoat' (Einstein 1973: 294). Like any other kind of theory, semantic theory is developed by applying the analyst's experience and intuitions to inferences drawn from occurrences of actual speech events to create a demonstrably rational account of their structures and causes.

'Semantic primitives', more recently called 'semantic primes', are the primitive symbols in a metalanguage for semantics, and their interpretations constitute the vocabulary of the semantic metalanguage. There was the search for semantic primes by Bishop John Wilkins (1668) in his Essay Towards a Real Character and a Philosophical Language. His contemporaries, Antoine Arnauld and Pierre Nicole in La logique, ou l'art de penser (1662) (Arnauld and Nicole 1965), recognised that the meanings of most words can be defined in terms of others, but that ultimately there are some undefinable semantically primitive words. 
In more recent times, Uriel Weinreich (1980: 50, 161, 300, 308-09) identified a three-step discovery procedure for a semantic metalanguage built upon natural language: (a) Stratify the language into a central core of semantic primitives whose members are definable only circularly and by ostensive definition such as 'colour of the sky' in the entry for blue. (b) The next stratum out uses items whose definitions contain only core items without (further) circularity. (c) Each more peripheral stratum uses items from the preceding strata without circularity. This is a goal that has not yet been achieved by anyone, though it is probably worth striving for.

Since before 1972, Anna Wierzbicka (see Wierzbicka 1972), influenced by Russian semanticists such as Apresjan $(1974,2000)$, has been carrying out this program in a cross-language context, searching for a universal set of semantic primitives expressed principally through the vocabulary of English (though see e.g. Peeters 2006). ${ }^{2}$ Goddard (1994: 12) (Principle VI) claims that 'any simple proposition' expressed in Natural Semantic Metalanguage (NSM) using any one natural language (e.g. English) will be expressible in NSM using any other language (e.g. Japanese). This embodies a claim that NSM is linguistically and culturally unbiased and that there is a heuristic or algorithm for translation, although in fact none has been published. The aims of NSM are consistent with what has been described above:

all languages share a universal core, both in their lexicon and in their grammar; a core which constitutes the bedrock of human understanding, communication, and translation. This shared core is like a mini-language, which can be used as a culturally neutral semantic metalanguage for the description of all languages, for the study of cultural diversity as well as the psychological unity of humankind, and also for applied purposes, in education and cross-cultural communication. (Wierzbicka 2006: 1)

Wierzbicka's search for semantic primitives recalls the 'Swadesh list' of basic vocabulary created to plot diachronic relationships between unwritten languages in Africa, the Americas and elsewhere. The purpose of the Swadesh list was to take a pair of languages and compare 100-215 basic lexemes to see how many are cognates (see Swadesh 1955); hence, one name for the program is 'lexico-statistics'. In making the comparisons,

2 A reviewer has commented that $s /$ he has heard Wierzbicka remark that her original semantic primitives were founded on her native Polish, the language in which what became Wierzbicka (1972) was first composed. 
literal meanings are preferred to semantic extensions; for example, the body-part sense of English tongue is preferred to the sense 'language'. Assuming that two languages being compared are in fact related, the time of divergence from a common mother language is estimated from the proportion of vocabulary common to both. The scale of vocabulary differentiation derives from studies of Indo-European languages for which there are historical records. The procedure is sometimes called 'glottochronology'. Words in the Swadesh list are basic in the sense that they name things likely to be common to the experience of all human communities, hence they fall into categories such as personal pronouns, interrogatives, connectives (and, if, because), locatives and locations, position and movement, manipulations (wash, hit, scratch), time periods, numerals, quantifiers, size, natural objects and phenomena, plants, animals, persons, body parts and substances, bodily sensations and activities, colours, kin and cultural objects and activities. There is clearly some similarity with the sets of semantic primes regularly set out in most works on NSM. However, the studies of semantic and lexical universals reported in Goddard and Wierzbicka (1994), Wierzbicka (1996), Goddard (1998) and many later works are not concerned with diachronic relationships, but with the different differential values that listemes have both within and across languages (a 'listeme' is a language expression whose meaning is not determinable from the meanings (if any) of its constituent forms and which, therefore, a language user must memorise as a combination of form and meaning: cf. Di Sciullo and Williams 1987).

The number of semantic primitives identified by Wierzbicka grew from 14 in 1972 to nearly 20 in 1985, 37 in 1994, 57 in 1998 and about 65 today. $^{3}$ In addition, 'allolexes' of these primitives are permitted: for example, ME for I, WHO and WHAT for SOMEONE and SOMETHING, THING for SOMETHING, COULD for CAN, FOR for BECAUSE, PLACE for WHERE. Exactly what constrains the proliferation of allolexes remains to be defined. And although NSM is sometimes said to be 'language-neutral' (Goddard 2001: 659), it is not. There are about 65 semantic primes in every language, such that there is an English NSM, a French NSM, a Mandarin NSM, a Tamil NSM, etc. It is claimed that the primes from one language correspond to those from any other language: 'they are isomorphic and constitute, in effect, different variants of one language-

3 See further discussion available at: intranet.secure.griffith.edu.au/schools-departments/naturalsemantic-metalanguage/what-is-nsm. This informative website is maintained by Cliff Goddard and colleagues. 
independent conceptual system' (Wierzbicka 2006: 3). However, NSM primes are compositionally and often semantically different across languages. Like most translated terms, the meanings show partial overlap rather than complete identity; for example, for NSM researchers, English SOME $=$ French IL Y A ... QUI; English THERE IS = French IL Y A. Yet French IL Y A UN X QUI is not equivalent to English THERE Is SOME $\mathrm{X}$ WHICH, where there is apparent translation equivalence between UN and sOME. Given that French IL Y A occurs within two primes, surely Un, the French equivalent to English prime ONE, could appear twice as well (cf. Peeters et al. 2006). Add to this that, in NSM, allolexes are not only tolerated but necessary, which makes the so-called 'semantic primes' more like meaning clusters than true primes; for example, English I and ME; DO, DOES, DID; French TU, TOI, vOUS; Italian TU, VOI, LEI, etc. Furthermore, primes are not independent of one another, there are several complementary pairs such as GOOD $\sim \mathrm{BAD}$, BIG $\sim$ SMALL, NEAR $\sim \mathrm{FAR}$, ABOVE $\sim \mathrm{BELOW}$, BEFORE $\sim$ AFTER, LIVE DIE. If NSM researchers were to stick rigidly to the notion of primes, such opposed pairs could each be reduced by one prime (the other being its negation) without thereby distorting natural language any more than is normal for the mini-language that is NSM. ${ }^{4}$ Indeed, until around 1990, Wierzbicka's meaning descriptions used a lot of other items besides the primes (cf. Wierzbicka 1972: 22, 26, 106). Most recent semantic analyses stick more closely to the primes.

Today, many NSM analyses contain 'semantic molecules', marked by a subsequent $[\mathrm{m}]$ (see (80-120) below for instances).

These are non-primitive meanings (hence, ultimately decomposable into semantic primes) that can function as units in the semantic structure of other, yet more complex words ... $[S]$ emantic molecules must be meanings of lexical units in the language concerned.

From a conceptual point of view, the NSM claim is that some complex concepts are semantically dependent on other less complex, but still non-primitive, concepts. For example, semantic explications for words like sparrow and eagle include 'bird' as a semantic molecule; the cognitive claim is that the concept of

4 A reviewer disputes this, citing objections in Wierzbicka (1996). I don't believe that there can be a restricted set of semantic primes such as those proposed in NSM; in my view, the number of semantic elements in any language is more or less the same size as its vocabulary. Furthermore, the proposed primes of NSM are rarely (if ever) monosemic. For instance, ABOvE is no more monosemic than is over in Brugman (1983), Dewell (1994), Lakoff (1987). 
sparrow includes and depends on the concept of 'bird'. In this case, the relationship is taxonomic: sparrows and eagles are both 'birds $[\mathrm{m}]$ of one kind' (molecules are marked in explications with the notation [m]). (Goddard 2010: 124)

Although it is said that all semantic molecules are reducible to semantic primes, this has only been demonstrated for a few (e.g. Goddard 2010: 125-130).

How do Anna Wierzbicka's semantic analyses compare to those of scholars using other metalanguages? In this essay I limit myself to critiquing her semantics for cup in Wierzbicka (1984). The expressions used in a semantic representation in NSM are supposed to match those that (a) children acquire early and (b) have counterparts in all languages (Goddard 1994: 12). In her definitions, Wierzbicka is deliberately anthropocentric and subjective, referring to the natural world of sensory experience rather than intellectualised abstractions. Thus, she prefers to describe red as the colour of blood (Wierzbicka 1980, 1990) or fire (Wierzbicka 1990, 1992a) than as an electromagnetic wave focally around 695 nanometres in length.

If we are trying to understand and to elucidate the intuitions of ordinary speakers, we cannot use in our definitions anything which is not independently attested to be accessible to that intuition. Translating colour terms into information about wavelength may tell us something about physiological and neurological processes but obviously it cannot tell us anything about the intuitive connections between different everyday concepts. (Wierzbicka 1984: 235)

I propose that blue involves more than one point of reference: not only the sky but also water-not water from the tap, but naturally occurring water, that is, the water of seas, lakes, rivers, and so on. Roughly:

$\mathrm{X}$ is blue

when one sees things like $\mathrm{X}$

one can think of the sky

or of places (not made by people)

where there is water

(Wierzbicka 1992b: 222-23)

This is a characteristic of cognitive semantics which I fully endorse. 
In the remainder of this essay I discuss Anna Wierzbicka's definition of cup from Wierzbicka (1984: 222-24). But, because this was published some 35 years ago I shall also briefly discuss Cliff Goddard's rather similar definition from Goddard (2011: 228-29) before considering alternatives. First, Wierzbicka's semantics from 1984, to which I have added paragraph numbers for the convenience of discussion.

(1) cup

(2) A KIND OF THING MADE BY PEOPLE

(3) IMAGINING THINGS OF THIS KIND PEOPLE WOULD SAY THESE THINGS ABOUT THEM:

(4) [Purpose]

(5) they are made for people to use repeatedly for drinking hot liquids from, such as tea or coffee

(6) one person from one thing of this kind

(7) being able to put them down on something else

(8) [Material]

(9) they are made of something rigid, smooth and easy to wash

(10) which liquids can't go into or pass through

(11) and which doesn't break easily in contact with hot liquid

(12) [Appearance: top]

(13) they are rounded and open at the top

(14) so that one can drink easily from them by tipping the top part slightly towards the mouth

(15) without any of the liquid going outside, where one doesn't want it to go

(16) [Appearance: bottom]

(17) the bottom is the same shape as the top

(18) so that they are not more difficult to make than they have to be and it is flat

(19) so that things of this kind can be put down on something else that is flat

(20) [Appearance: proportions]

(21) they cannot be much wider than they are high 
(22) so that the liquid inside doesn't cease to be hot before one can drink it all

(23) they cannot be much higher than they are wide

(24) so that they don't overturn easily when one puts them down somewhere

(25) [Size]

(26) they have to be big enough to be able to have not less hot liquid in

(27) than a person would be expected to want to drink of that kind of liquid at one time

(28) they cannot be too big for people to be able to raise them easily to the mouth full of liquid, with one hand

(29) IMAGINING THINGS OF THIS KIND PEOPLE COULD ALSO SAY THESE THINGS ABOUT THEM:

(30) [Use]

(31) people drink from them when sitting at a table

(32) [Use: sets]

(33) they are made and used in groups of things which look the same

(34) so that they look nice together

(35) [Use: saucers]

(36) they are made and used together with some other things

(37) made of the same stuff

(38) made for putting these things on

(39) one thing for a person to drink from, on one thing for putting such things on

(40) so that people can raise the things to drink from to the mouth to drink a little

(41) and then put them down on those other things which can be put down on the table

(42) those other things are similar to them in some ways so that they look nice together

(43) those other things are made in such a way that there can be some parts of them all around the bottom of the things that people drink from

(44) so that if any liquid goes down over the top of the things people drink from

(45) it will come onto and remain on those other things 
(46) and will not get elsewhere, where one doesn't want it to go

(47) [Material]

(48) things of this kind are made of something thin so that they are nice to look at and to drink from

(49) and of something that one can't see through

(50) so that things made of it can have pictures and patterns on them

(51) making them nice to look at,

(52) such as china

(53) [Appearance: sides]

(54) the sides are rounded, not flat

(55) so that all the sides look the same and one can't say where one side ends and another begins

(56) so that one can easily hold things of this kind around with a hand when they don't have any hot liquid inside

(57) the sides are not straight but roundish

(58) so that looked at from the side the opposite sides, or their upper parts, look like rounded lines

(59) whose middle parts are further away from one another than their tops or bottoms

(60) [Appearance: proportions]

(61) the bottom is smaller than the top

(62) so that if some of the liquid goes down along the outer surface of the thing one is drinking from

(63) it will come to the middle of the thing it is on

(64) and will not go outside that thing to where one doesn't want it to go

(65) [Appearance: handle]

(66) things of this kind have a thin looped part for holding

(67) which sticks out from one side

(68) which doesn't get hot

(69) because it is not in contact with the hot liquid inside

(70) this part is attached at its top and its bottom to one side

(71) so that by holding it one can prevent one part of the top from being above the others when one is not drinking 
(72) and so prevent any of the liquid from getting out over the top and going where one doesn't want it to go

(73) this part has to be big enough and to stick out far enough for people to be able to raise things of this kind full of liquid for a short time

(74) holding that part with a thumb and two fingers

(75) without any parts of the hand touching the sides, which are hot

(76) it can't be much bigger or stick out much further than necessary for that. (Wierzbicka 1984: 222-24)

One of the most striking things about this definition of cup is its detail and consequently its length (about 830 words). A second is that it includes far more than a vocabulary of primes. I will discuss first the detail and length and turn to the exoticism after exemplifying Goddard's version, which sticks more closely to semantic primes and marks semantic molecules.

(2) identifies a cup as a manufactured, not natural, object. (3) anthropocentrically identifies the content as cognitively real. It is unclear to me why (29) is needed, but apparently characteristics (30-76) are seen as secondary elaborations on (2-28) and, in fact, many of them do seem redundant. A cup's purpose, (4-7), and the function ((30-46)) for which this artefact is manufactured will (at least partially) determine the material from which it is made, $(8-11,47-52)$, and its configuration, (12-28, 53-76). With respect to function: although it is true that cups are primarily for the drinking of hot liquids (which is probably why they typically have handles, (65-76)), cups are also used for cool and cold liquids and for measuring dry goods such as flour and sugar; given the amount of detail in Wierzbicka's description it is surprising that such secondary uses are disregarded.

(8-11) identify a material necessary for containing potentially hot liquid. There is no mention of semantic extensions such as single use paper and plastic cups (which typically lack handles).

Cups are very possibly modelled on a human's cupped hands. A typical cup $^{5}$ holds around 250 millilitres, which is similar to the capacity of adult male cupped hands. A single hand cupped holds around $125 \mathrm{~mL}$, roughly equivalent to the amount of liquid held by an espresso coffee cup

5 I prefer the term typical to prototypical or stereotypical for reasons explained in Allan (2001: 334-36). 
or a Middle Eastern tea or coffee cup-which is bowl-like (i.e. handleless). Thus, a typical cup is a hollow oblate hemispheroid ${ }^{6}$ container (i.e. a squashed half-sphere) with a flat base at the pole (its base or bottom) so that it can easily stand alone, open at the wide end for easy access by human lips to the liquid it contains. It is designed to be readily manipulated by the thumb and fingers of a single human hand. These matters of configuration are over-elaborated in (12-28). A rectangular cup would be atypical because impracticable, but nonetheless it could function as a cup, because the essential criterion for a cup is that it be a container for liquid with a capacity of around $250 \mathrm{ml}$.

Consequently, (31) seems to me to be irrelevant: what's criterial for a cup is that it is a container that serves as a drinking vessel which can be handled by children as well as adults, so it must be lightweight. Whether the user is sitting, standing or lying in bed, is irrelevant. It is also as irrelevant to the meaning of cup that cups often come in sets, (32-34), as that they come in a variety of colours and designs - and there is (correctly) nothing said about those characteristics in (1-76).

(35-46) over-elaborate the significance of saucers. Typically cups in the West are accompanied by saucers-though Middle-Eastern cups typically are not. The saucer is a practical stand for a cup, etiquette favours their use as practical protectors of clothing and furniture from hot cups and dribbled liquid; they may also be used to park a teaspoon or used tea-bag. Mugs are typically cylindrical drinking vessels that don't have saucers. ${ }^{7}$

(47-52) are a matter of aesthetics irrelevant to the meaning of cup. (53-64) are unnecessary aspects of the configuration of cups that has already been adequately covered in (12-28).

(65-76) identify a significant characteristic of (Western) cups: typically, they sport handles, $(66,67,70,76)$, to facilitate the conveyance of a cup of hot liquid to the mouth without discomforting the hand, $(69,75)$. Normally only the thumb and one or two fingers manipulate the handle,

6 Both cupped hands and a single cupped hand are similar in shape to a hollow oblate hemispheroid (if we ignore the attached arm). A tapered mug might be described as a hollow prolate hemispheroid, though most mugs are cylindrical.

$7 \quad$ Wierzbicka (1984) and Goddard (1998, 2011), written in response to Labov (1973), specifically contrast cups with mugs, which there is no space to do here, though I have done so in Allan (1986/2014, 2001). 
(74). Typical Middle Eastern tea and coffee cups are small enough to be held by the thumb and first finger, often around a rim that does not overheat. Typical single-use paper or plastic cups lack handles and saucers.

Let me now introduce Cliff Goddard's semantics for cup. In Goddard (1998: 233) he offered a somewhat shorter version than in his revised version in 2011 to which I have, for convenience in discussion, added numbers $(77-120)$.

(77) a cup:

FUNCTIONAL CATEGORY

(79) a. something of one kind

(80) at many times people do something with something of this kind when they are drinking $[\mathrm{m}]$ something hot $[\mathrm{m}]$

(81) when someone is drinking [m] something like this, before it is inside this someone's mouth [m], it is for some time inside something of this kind

SIZE

(83) b. things of this kind are like this:

(84) - they are not big

(85) - someone can hold [m] one in one hand [m]

(87) many things of this kind have a small thin [m] part on one side

(88) when someone is drinking [m], this someone can hold [m] this part with the fingers $[\mathrm{m}]$ of one hand $[\mathrm{m}]$

OTHER PARTS

(90) the other parts are like this:

(91) - the sides [m] are like the sides [m] of something round [m]

(92) - they are thin [m]

(93) - the top [m] part of the sides has a smooth [m] round [m] edge $[\mathrm{m}]$

(94) - the bottom [m] part of something of this kind is flat [m]

(95) - someone can think that the bottom [m] part is small, if this someone thinks about the top $[\mathrm{m}]$ part at the same time 
(97) things of this kind are made of [m] something hard [m]

(98) this something is smooth [m]

(100) c. when someone is doing something with something of this kind because this someone is drinking $[\mathrm{m}]$ something hot $[\mathrm{m}]$, it happens like this:

(101) - at some time this something is in one place for some time, at this time the bottom $[\mathrm{m}]$ part is touching something flat $[\mathrm{m}]$

(102) - at this time there is something like hot $[\mathrm{m}]$ water $[\mathrm{m}]$ inside this thing

(103) - it can be tea [m], it can be coffee [m], it can be something of another kind

(104) - it is inside this thing because some time before someone did some things because this someone wanted it to be like this

(105) - after this, someone picks up [m] this something with the fingers $[\mathrm{m}]$ of one hand $[\mathrm{m}]$

(106) - after this, this someone does something else to it with the hand $[\mathrm{m}]$

(107) - after this, because of this, part of the edge [m] at the top [m] of this thing touches one of this someone's lips [m] for a short time, as this someone wants

(108) - during this time, this someone's fingers [m] move as this someone wants

(109) - because of this, a little bit of something like hot [m] water [m] moves, as this someone wants

(110) - because of this, after this it is not inside this thing anymore, it is inside this someone's mouth [m]

(111) - after this, this someone puts [m] this thing down [m] on something flat $[\mathrm{m}]$

(112) - after this, this someone can do this a few more times

SAUCER

(114) sometimes when someone is drinking [m] something in this way, this someone wants not to hold $[\mathrm{m}]$ this thing for a short time 
(115) when it is like this, this someone can put [m] this thing down [m] on something of another kind, in the middle $[\mathrm{m}]$ of this other kind of thing

(116) these other things are made of [m] the same hard [m], smooth [m] stuff

(117) they are round $[\mathrm{m}]$, they are flat $[\mathrm{m}]$

(118) the edge [m] of something or this kind is above the middle [m] ARTEFACT STATUS

(120) d. many people want to drink [m] things of some kinds like this at many times because of this, some people make [m] things of this kind. (Goddard 2011: 228-29)

A few general remarks: the principal contrast with Wierzbicka's account is that Goddard's sticks much more closely to semantic primes, despite the extensive employment of 'semantic molecules' marked by a subsequent [m]. It is also a little shorter, 66 paragraphs instead of 76. In my view it is still far too long because it includes some extraneous information while omitting some criterial information. It is sectioned into four parts: (a), (78-81), identifies a cup's primary function; (b), (82-98), describes the configuration of a typical cup and the material from which it is made; (c), (99-118), describes how a cup is used and what it is used for, then brings in saucers; and (d), (119-120), says the cups are in wide use and many are manufactured.

(78-81) identify a cup as, primarily, a vessel for containing hot liquid. (82-95) identify the typical configuration: a cup can be held by the fingers of one hand, for which reason it has a handle; it is a hollow oblate hemispheroid with a flat bottom. (96-98) describe the material from which a cup is made as smooth and hard - which doesn't absolutely exclude single-use paper or plastic cups, and does recognise the character of a typical cup. (99-112) describes the use of cup for the drinking of hot liquid, mentioning that a cup is several times raised to the lips and lowered onto a flat surface; although commonly true, this is superfluous information because it has no part in defining what a cup is. (113-118) describes the configuration and constituency of a saucer but doesn't offer a satisfactory account of its function. Finally, (119-120) says that because people like drinking hot liquids, cups are manufactured to that purpose. 
So, the Goddard (2011) semantics for cup is inspired by his mentor Wierzbicka's earlier account and, as an exercise in NSM, greatly improves on it by sticking quite closely to the use of semantic primes while marking digressions into semantic molecules that stray from primes by invoking complexes of primes, though no explication of these complexes is presented. Wierzbicka (1984) was explicitly a refutation of Labov's denotation conditions for cup (Labov 1973: 366f), on the grounds that they 'need the help of a mathematician to understand' them and do not give the lexicographic meaning (Wierzbicka 1984: 207). She claims 'the denotation conditions can be deduced from the meaning' (1984: 209). So, let's review Labov's denotation conditions:

The term cup is regularly used to denote round containers with a ratio of width to depth of $1 \pm \mathrm{r}$ where $\mathrm{r} \leq \mathrm{r}_{\mathrm{b}}$, and $\mathrm{r}_{\mathrm{b}}=\alpha_{1}+\alpha_{2}+\ldots \alpha_{v}$ and $\alpha_{1}$ is a positive quantity when the feature $i$ is present and 0 otherwise.

feature 1 = with one handle

$2=$ made of opaque vitreous material

$3=$ used for consumption of food

$4=$ used for consumption of liquid food

$5=$ used for consumption of hot liquid food

$6=$ with a saucer

7 = tapering

$8=$ circular in cross-section

Cup is used variably to denote such containers with ratios of width to depth of $1 \pm r$ where $r_{b} \leq r \leq r_{t}$ with a probability of $r_{t}-r / r_{t}-r_{b}$. The quantity $\mathrm{r} \pm \mathrm{r}_{\mathrm{b}}$ expresses the distance from the modal value of width to height. (Labov 1973: 366f, quoted in Wierzbicka 1984: 206f)

Labov's is notably succinct by comparison with the versions of Wierzbicka (1-76) and Goddard (77-120) and I do not find it more troublesome to read through than the NSM versions. An important question arises about the playoff between the effectiveness of a definition and its accuracy. What is the purpose of the semantic analysis? Who or what is the semantic specification that results from the analysis designed for? Wierzbicka's semantic definitions are not designed to be used by machines that simulate language understanding. She intends them to be easily accessible to a nonnative speaker of the language. But every such reader will already know what a cup is, so a brief description would be sufficient. In my experience, 
students find Wierzbicka's two-page definition of cup just as challenging as Labov's denotation conditions. Consider the following 'dictionary representation' of cup given by Jerrold J. Katz:

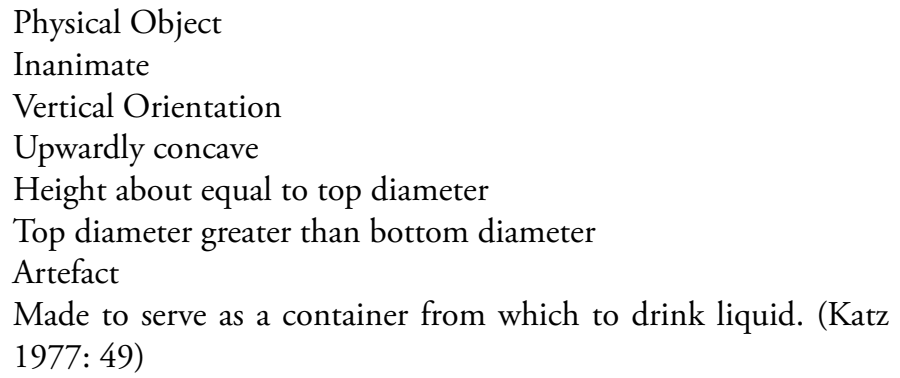

Katz's description is adequate, very much simpler than Labov's 'denotation conditions', and much more succinct than Wierzbicka's 'semantic definition' of c. 830 words.

If anything, the Oxford English Dictionary $(O E D)$ is simpler still:

1. A small open vessel for liquids, usually of hemispherical or hemi-spheroidal shape, with or without a handle; a drinkingvessel. The common form of cup (e.g. a tea-cup or coffee-cup) has no stem; but the larger and more ornamental forms (e.g. a winecup or chalice) may have a stem and foot, as also a lid or cover; in such case cup is sometimes applied specifically to the concave part that receives the liquid.

But as will be seen in (121), I believe that even this august lexical entry does not fully specify what a cup is.

Wierzbicka and Goddard might object that the terms Katz and the OED use are more difficult than cup itself (which is arguably equally true for the semantics of cup in Wierzbicka 1984 and Goddard 1998, 2011); this however is irrelevant to proper statement of the meaning. The purpose of semantic representations is to make useful revelations about the nature of human language and/or human cognition. Wierzbicka has written:

An adequate definition must show fully what the word in question means, not what it doesn't mean or how it differs in meaning from some other words which we happen to compare it with. ... [A]n adequate definition of a word must constitute a faithful 'portrait' of the concept encoded in it. (Wierzbicka 1984: 227) 
This is inconsistent with her semantic definition of tiger (Wierzbicka 1985: 164), where she compared tigers with cats because we see tigers as a kind of cat. In different terms, what Wierzbicka seems to be saying is that an adequate definition of what a listeme means will capture the intension of the listeme-which is exactly my own view. And the complexity of explication is unimportant provided it is accurate and revealing-as should be obvious from perusal of entries in the $O E D$.

At this point it behoves me to offer my own version of the semantics for сир (and, like Wierzbicka and Goddard, I restrict this to the drinking vessel, ignoring such similarly shaped objects as acorn cups and bra cups). On this occasion, I use English as an informal metalanguage.

(121) cup

Hollow oblate hemispheroid vessel, flat at its pole, with a capacity $\approx 250 \mathrm{ml}(\approx$ the capacity of cupped human hands) and a vertical handle to facilitate drinking when held by a human's thumb and one or two fingers in order to raise the vessel to the lips. Typically used for hot liquids. Often accompanied by a saucer to stand on. Middle Eastern cups typically have half the capacity of Western cups and, like single-use paper and plastic cups, they typically lack handles and saucers.

The prolixity of Wierzbicka's and Goddard's semantic definitions for cup is unjustified. Although NSM authors do not specifically create lexicon entries, such seem an appropriate application for the lexical analyses on offer-especially given that Goddard (2010: 124) writes of developing 'our picture of the overall structure of the lexicon'. As Alan Cruse (1990: 396) wrote: 'For dictionary purposes, the concept has only to be identified, not fully specified.' Nonetheless, (121) is a fuller specification than in any of the other semantics presented for cup that I have exemplified and discussed, and it clearly identifies the concept of a cup. Hopefully, you, the reader, will agree.

\section{Acknowledgement}

I would like to thank two anonymous referees for useful comments that led to an improved text. However, no one but me is responsible for the infelicities that remain. 


\section{References}

Allan, Keith (1986/2014). Linguistic Meaning (2 vols). London: Routledge and Kegan Paul. [Reprint edn, (1991) Beijing: World Publishing Corporation. Reissued in one volume as Routledge Library Editions: Linguistics Volume 8 (2014).]

Allan, Keith (2001). Natural Language Semantics. Oxford and Malden, Massachusetts: Blackwell.

Apresjan, Juri D. (1974). Regular polysemy. Linguistics 142: 5-32.

Apresjan, Juri D. (2000). Systematic Lexicography (trans. by Kevin Windle). Oxford: Oxford University Press.

Arnauld, Antoine and Pierre Nicole (1965). La Logique, ou L'art de Penser: contenant, outre les règles communes, plusieurs observations nouvelles propres à former le jugement [Logic or The Art of Thinking: Containing, Besides Common Rules, Several New Observations Appropriate for Forming Judgment]. Éd. critique par Pierre Clair et François Girbal [critical edition by Pierre Clair and François Girbal, based on the final 1683 version]. Paris: Presses universitaires de France.

Brugman, Claudia M. (1983). The Story of Over. Bloomington: Indiana University Linguistics Club.

Carnap, Rudolf (1937). Logical Syntax of Language. London: Routledge and Kegan Paul.

Cruse, D. Alan (1990). Prototype theory and lexical semantics. In Savas L. Tsohatzidis (ed.), Meanings and Prototypes: Studies in Linguistic Categorization. London: Routledge, 382-402.

Dewell, Robert B. (1994). Over again: Image-schema transformations in semantic analysis. Cognitive Linguistics 5: 351-80. doi.org/10.1515/cogl.1994.5.4.351.

Di Sciullo, Anna-Maria and Edwin Williams (1987). On the Definition of Word. Cambridge, MA: MIT Press.

Einstein, Albert (1973 [1954]). Ideas and Opinions (Laurel edn). New York: Dell.

Goddard, Cliff (1994). Semantic theory and semantic universals. In Cliff Goddard and Anna Wierzbicka (eds), Semantic and Lexical Universals: Theory and Empirical Findings. Amsterdam: John Benjamins, 7-30.

Goddard, Cliff (1998). Semantic Analysis: A Practical Introduction. Oxford: Oxford University Press. 
Goddard, Cliff (2001). Sabar, iklas, setia-patient, sincere, loyal? Contrastive semantics of some 'virtues' in Malay and English. Journal of Pragmatics 33: 653-81. doi.org/10.1016/s0378-2166(00)00028-x.

Goddard, Cliff (2010). Semantic molecules and semantic complexity (with special reference to 'environmental' molecules). Review of Cognitive Linguistics 8 (1): 123-55. doi.org/10.1075/ml.8.1.05god.

Goddard, Cliff (2011). Semantic Analysis: A Practical Introduction (2nd edn). Oxford: Oxford University Press.

Goddard, Cliff and Anna Wierzbicka (eds) (1994). Semantic and Lexical Universals: Theory and Empirical Findings. Amsterdam: John Benjamins.

Katz, Jerrold J. (1977). A proper theory of names. Philosophical Studies 31: 1-80.

Labov, William (1973). The boundaries of words and their meanings. In Charles-J. Bailey and Roger Shuy (eds), New Ways of Analyzing Variation in English. Washington DC: Georgetown University Press, 340-73.

Lakoff, George (1987). Women, Fire, and Dangerous Things: What Categories Reveal About the Mind. Chicago: University of Chicago Press.

Oxford English Dictionary (OED) (n.d.). Oxford: Oxford University Press. Available at: www.oed.com/.

Peeters, Bert (ed.) (2006). Semantic Primes and Universal Grammar: Empirical Evidence from the Romance Languages. Amsterdam: John Benjamins. doi.org/ $10.1075 /$ slcs. 81 .

Peeters, Bert, Marie-Odile Junker, Patrick Farrell, Pedro Perini-Santos and Brigid Maher (2006). NSM exponents and universal grammar in Romance: Speech; actions, events and movement; existence and possession; life and death. In Bert Peeters (ed.), Semantic Primes and Universal Grammar: Empirical Evidence from the Romance Languages. Amsterdam: John Benjamins, 111-36. doi.org/10.1075/slcs.81.11pee.

Swadesh, Morris (1955). Towards greater accuracy in lexico-statistic dating. International Journal of American Linguistics 21: 121-37. doi.org/10.1086/ 464321 .

Weinreich, Uriel (1980). Weinreich on Semantics (ed. by William Labov and Beatrice S. Weinreich). Philadelphia: University of Pennsylvania Press.

Wierzbicka, Anna (1972). Semantic Primitives. Frankfurt: Athenäm.

Wierzbicka, Anna (1980). Lingua Mentalis: The Semantics of Natural Language. Sydney: Academic Press. 
Wierzbicka, Anna (1984). Cups and mugs: Lexicography and conceptual analysis. Australian Journal of Linguistics 4: 257-81. doi.org/10.1080/ 07268608408599326 .

Wierzbicka, Anna (1985). Lexicography and Conceptual Analysis. Ann Arbor: Karoma.

Wierzbicka, Anna (1990). The meaning of color terms: Semantics, culture, and cognition. Cognitive Linguistics 1:99-150. doi.org/10.1515/cogl.1990.1.1.99.

Wierzbicka, Anna (1992a). Semantic primitives and semantic fields. In Eva Kittay and Adrienne Lehrer (eds), Frames, Fields, and Contrasts. Norwood, NJ: Lawrence Erlbaum, 209-27.

Wierzbicka, Anna (1992b). Personal names and expressive derivation. In Semantics, Culture, and Cognition: Universal Human Concepts in Culturespecific Configurations. New York: Oxford University Press, 225-307.

Wierzbicka, Anna (1996). Semantics: Primes and Universals. Oxford: Oxford University Press.

Wierzbicka, Anna (2006). Preface. In Bert Peeters (ed.) Semantic Primes and Universal Grammar: Empirical Evidence from the Romance Languages. Amsterdam: John Benjamins, 1-6. doi.org/10.1075/slcs.81.05wie.

Wilkins, John (1668). Essay Towards a Real Character and a Philosophical Language. London: S. Gellibrand and John Martin for the Royal Society [Menston: Scolar Press Facsimile. 1968]. 
This text is taken from Meaning, Life and Culture: In conversation with Anna Wierzbicka, edited by Helen Bromhead and Zhengdao Ye, published 2020 by ANU Press, The Australian National University,

Canberra, Australia.

doi.org/10.22459/MLC.2020.23 